\title{
CONFERENCE AND EXHIBITION ORGANIZERS
}

\author{
BIS APPLIED SYSTEMS LTD \\ York House \\ 199 Westminster Bridge Road \\ London SE1 7NT \\ Telephone: 016330866 \\ Telex: 919642 BISDAT G
}

\section{CGS INSTITUTE}

Russell House

Russell Street

Windsor

Berkshire SL4 1HQ

Telephone: 0753858811

Telex: 849105

\author{
CABLE \& SATELLITE TELEVISION EXHIBITIONS \\ LTD \\ 3 Barratt Way \\ Tudor Road \\ Harrow \\ Middlesex HA3 5QG \\ Telephone: 018614877 \\ Telex: 297171 CAST G
}

\section{CHARVIL LEWIS INTERNATIONAL}

27 Marlow Road

Maidenhead

Berks

Telephone: 062874274

Telex: 847660 COLMAN G

\section{COMMUNICATIONS EDUCATIONAL SERVICES}

Communications House

137 Dulwich Road

London SE24 0NG

Telephone: 017333456

\section{EOSYS LIMITED}

Clove House

The Broadway

Farnham Common

Slough

Berks SL2 3PQ

Telephone: 028145123

Telex: 849826 EOSYS G

\author{
EUROPEAN STUDY CONFERENCES LTD \\ 31 High Street East \\ Uppingham \\ Rutland LE15 9PY \\ Telephone: 0572822711 \\ Telex: 341352 EURCON G \\ THE FINANCIAL TIMES CONFERENCE \\ ORGANISATION \\ Minster House \\ Arthur Street \\ London EC4R 9AX \\ Telephone: 016211355 \\ Telex: 27347 FTCONF G \\ FROST \& SULLIVAN LTD \\ 104-112 Marylebone Lane \\ London W1M 5FU \\ Telephone: 019353190 \\ Telex: 261671 FANDS G
}

\author{
INFORMATION TECHNOLOGY \& MARKETING \\ E3 New Enterprises \\ South West Brunswick Docks \\ Liverpool \\ Telephone: 0517089066 \\ INSTITUTE FOR INTERNATIONAL RESEARCH \\ 44 Conduit Street \\ London W1R 9FB \\ Telephone: 014341017 \\ Telex: 946240 CWEASY G \\ MAPLE COMMUNICATIONS MANAGEMENT \\ LIMITED \\ 500 Chesham House \\ 105 Regent Street \\ London W1R 5FA \\ Telephone: 014396288 \\ Telex: 849021 FRAN G \\ ONLINE INTERNATIONAL LTD \\ Pinner Green House \\ Ashdown Drive \\ Pinner HA5 2AE \\ Telephone: 018684466
}

OYEZ SCIENTIFIC \& TECHNICAL SERVICES LTD

Bath House (3rd floor)

56 Holborn Viaduct

London EC1A 2EX

Telephone: 012364080

Telex: 888870 